\title{
Gingival biotype: The probe test utility
}

\author{
Madline Cuny-Houchmand ${ }^{1}$, Stéphane Renaudin ${ }^{1}$, Mustapha Leroul ${ }^{1}$, Lucie Planche², \\ Laurent Le Guehennec ${ }^{3}$, Assem Soueidan ${ }^{4,5 *}$
}

${ }^{1}$ Department of Orthodontics, UFR d'Odontologie, Nantes, France

${ }^{2}$ Cellule de Promotion de la Recherche Clinique, CHU, Nantes, France

${ }^{3}$ Department of Prosthodontics, UFR d'Odontologie, Nantes, France

${ }^{4}$ Department of Periodontology, UFR d'Odontologie, Nantes, France

${ }^{5}$ UIC Odontologie, CHU, Nantes, France

Email: ${ }^{\text {assem.soueidan@,univ-nantes.fr }}$

Received 22 March 2013; revised 22 April 2013; accepted 7 May 2013

Copyright (C) 2013 Madline Cuny-Houchmand et al. This is an open access article distributed under the Creative Commons Attribution License, which permits unrestricted use, distribution, and reproduction in any medium, provided the original work is properly cited.

\begin{abstract}
Purpose: To evaluate the relevance of the probe test in classifying the gingival biotype and to determine if differences exist between maxillary and mandibular gingival biotypes in the same patient. Materials and Methods: 53 subjects were included. The gingival thickness was evaluated as thick or thin. This evaluation was based on the transparency of the periodontal probe through the gingival margin while probing the sulcus. In another hand, 124 clinicians were asked to identify the gingival biotype in the same 53 subjects based on visual inspection on pictures. For each examiner, the accuracy in identifying the gingival biotype was assessed using the percentage of cases correctly identified. Results: the percentile of agreement is greater than $\mathbf{7 0} \%$ in maxilla as well as in mandible. An imbalance was observed on the maxillary pictures concerning the gingival biotype and on the thin biotype between maxilla and mandible despite there being no statistical difference $(p<\mathbf{0 . 0 0 0 1})$. The percentile of agreement is quite similar in the mandible for both thin $(74 \%)$ and thick $(70 \%)$ gingival biotypes. Agreement between mandibular probe and maxilla probe results demonstrates a weak percentile of agreement of gingival biotypes between maxillary and mandibular anterior teeth $(57 \%)$. Conclusion: The use of probe transparency through the gingival margin is an accurate and simple method for gingival biotype diagnosis to discriminate a thin gingival from a thick gingival. Furthermore, individualizing the gingival biotype for each of the arches appears to be a new outcome that needs further investigation.
\end{abstract}

"Corresponding author.
Keywords: Probe; Gingival Biotype; Prevention; Classification

\section{INTRODUCTION}

During orthodontic treatment, periodontal attachment loss and marginal tissue recession are adverse effects that unfortunately are observed in patients presenting reduced gingival thickness. Diagnosing these patients when beginning an orthodontic treatment may help practitioners in making appropriate decisions concerning the degree of incisor inclination or intrusion. Nevertheless, due to several complex established classifications identification and categorization of gingival biotypes may appear difficult for clinicians. A relevant, reproducible, sensitive, predictive, and easy-to-use classification method has to be developed to easily detect and diagnose high-risk patients in terms of marginal tissue recession prior to orthodontic treatment. In a previous article [1], we tested a visual inspection method and our conclusions, consistent with the literature, demonstrated that visual inspection alone is not a reliable method for gingival biotype diagnosis. This study also demonstrates the existence of a different biotype between maxilla and mandible in a patient, requiring a specific diagnosis between the two arches.

The thin gingival biotype is usually characterized by a delicate soft tissue curtain, a scalloped underlying osseous form, and often presents bone dehiscence or fenestrations. The quantity and quality of keratinized mucosa is usually reduced. On the other hand, the thick gingival biotype covers up the thick buccal alveolar bone. The mucosa is fibrotic and resilient with a tendency for pocket formation rather than recession [2-4]. A new methodology has been recently developed by De Rouck et al. [4] for discriminating thin and thick gingival biotypes using 
a periodontal probe through the gingival margin. This simple visual method is based on the transparency of the periodontal probe through the gingival margin while probing the buccal sulcus at the midfacial aspect of both central maxillary incisors. When the outline of the underlying periodontal probe can be seen through the gingival, the gingival biotype is considered thin. The gingival biotype is thick in the other case. This methodology was originally developed for maxillary examinations; nevertheless, for orthodontics the risk of development of dehiscence is more important during the labial movement of the mandibular incisors. Moreover, several animal and human studies $[5,6]$ have shown that gingival thickness may vary between maxillary and mandibular arches, depending on gender and age. Due to these parameters, determination of only the maxillary biotype does not appear to be sufficient for discriminating periodontal risk patients.

The purpose of this study is to evaluate the relevance of the probe test in classifying the gingival biotype and to determine if differences exist between maxillary and mandibular gingival biotypes. The final goal is the risk identification and the prevention of tissular iatrogenic damages.

\section{MATERIALS AND METHODS}

\subsection{Subjects}

This study included 53 subjects ( 25 females and 28 males) with a mean age of 17.5 years. Thirty-six were volunteer dental students from the Nantes Dental Surgery Faculty, and 17 were patients receiving clinical orthodontic treatment in the Conservative and Paediatric Clinic at Nantes Hotel-Dieu Hospital. All subjects gave their informed consent to participate in this study.

The inclusion criteria were as follows:

- Subjects presenting all maxillary and mandibular incisors;

- Subjects presenting good oral hygiene without any clinical signs of gingival inflammation or attachment loss (periodontal probing does not exceed $3 \mathrm{~mm}$ ).

The exclusion criteria were as follows:

- Subjects with crown restorations or fillings that involved the incisal edge on anterior teeth;

- Pregnant or lactating women; and

- Subjects taking medication with any known effect on periodontal soft tissues.

\subsection{Clinical Parameters}

The gingival biotype for each of the subjects was classified by two experienced clinicians following the method described by De Rouck et al. [4]. Specifically, the clinicians' classification was called the "reference". These clinicians were trained in periodontology (FL) and orthodontia (LM).

The following two clinical parameters were recorded by these two examiners:

- Gingival thickness was evaluated as thick or thin. This evaluation was based on the transparency of the periodontal probe (HU-Friedy) through the gingival margin while probing the sulcus at the midfacial aspect of the four central incisors (Figure 1). If the probe could be seen, it was categorized as thin; if not, it was categorized as thick.

- Probing depth was measured at the midfacial aspect of maxillary and mandibular incisors.

Finally, for each patient two clinical pictures were taken using a Nikon D60 in macro mode with flash and a magnification of 0.5 (a total of 101 relevant pictures were taken). The first picture focused on the right maxillary incisor with the probe inserted in the sulcus, and the second picture focused on the right mandibular incisor under the same conditions.

\subsection{Visual Inspection by Clinical Staff}

124 clinicians of the Dental Surgery Faculty at Nantes were asked to identify the gingival biotype in the same 53 subjects based on visual inspection alone. Among the practitioners, nine were specialists in orthodontia (i.e., group C) and nine were residents in Dental Surgery (i.e., group I). Moreover, 48 were dental students in the final year of graduate program (i.e., group $\mathrm{T}$ ) and the remaining 58 were inexperienced dental students beginning their studies (i.e., group D).
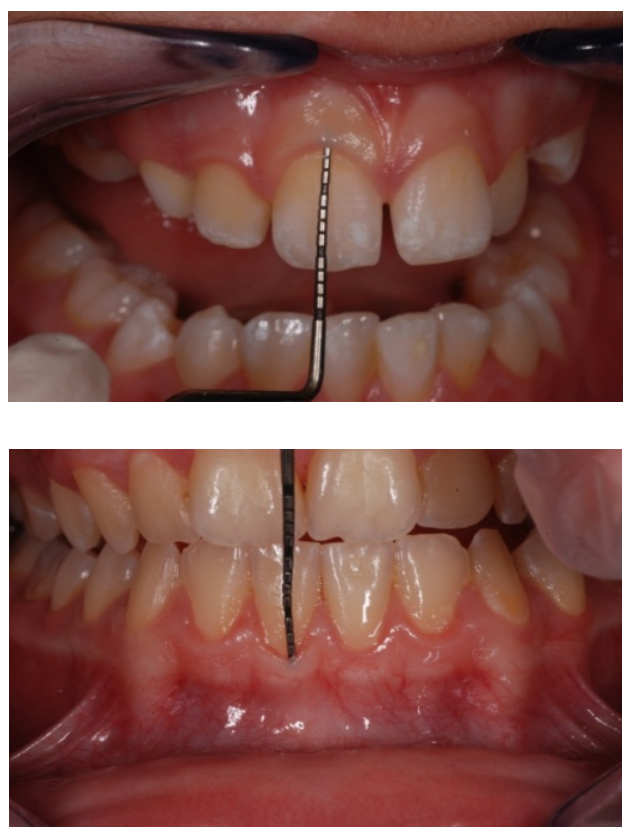

Figure 1. Probe test on maxillary and mandibular central incisors. 


\subsection{Scoring}

Examiners were installed in a dark room. Explanations for the cluster analysis in the two groups (thin or thick in relation to visualization of the probe through the margin) were given by a periodontist (AS). The transparency of the gingival margin was tested on 106 pictures/slides projected in a randomized order: 48 slides with the probe in the midfacial aspect of the maxillary incisor and 53 with the probe in the midfacial aspect of the mandibular incisor. The clinicians had to answer yes if they could see the probe and no if they could not. Each slide was projected for 8 seconds on a large screen.

Responses were collected and entered into Excel for statistical analysis.

\subsection{Statistical Analysis}

- Accuracy in identifying the gingival biotype

The accuracy in identifying the gingival biotype was evaluated for the lower and upper arches and compared with De Rouck classifications. For each examiner, the accuracy in identifying the gingival biotype was assessed using the percentage of cases correctly identified. The overall average percentage of cases correctly identified and the average percentage of cases correctly identified by a type of examiners were estimated with a $95 \%$ confidence interval. The overall average percentage of cases correctly identified in each case was tested using paired Student $t$ test. The accuracy of identifying each of the two gingival biotypes (thin/thick) was detailed.

- Agreement between lower and upper arches

The agreement was first analyzed between the mandibular reference and the maxillary reference using percentage of agreement and Kappa Statistics with a 95\% confidence interval.

The agreement was also evaluated between mandible and maxilla.

For each examiner, percentage of agreement and kappa statistics were determined. The overall average percentage of agreement and the average percentage of agreement by type of examiners were estimated with a $95 \%$ confidence interval. The overall agreement was evaluated using the mean of kappa statistics with a $95 \%$ confidence interval.

\section{RESULTS}

In order to evaluate the reliability of probe inspection, we have calculated the number of cases when each clinician has correctly identified the probe in both maxilla and mandible.

\subsection{References' Classification Results (Table 1)}

On the mandible, the probe appeared in transparency on
Table 1. References' classification results.

\begin{tabular}{ccccc}
\hline \multirow{2}{*}{ Maxilla } & \multicolumn{3}{c}{ Mandible } \\
\cline { 3 - 5 } & & Thin & Thick & Total \\
\hline \multirow{3}{*}{ Thin } & 4 & 6 & 10 \\
& Thick & 19 & 24 & 43 \\
& Total & 23 & 30 & 53 \\
\hline
\end{tabular}

23 pictures (thin biotype) and was not visible on the 30 residual pictures (thick biotype).

On the maxilla, the probe appeared in transparency on 9 pictures (thin biotype) and was not visible on the 39 residual pictures (thick biotype).

\subsection{Accuracy of Mandible and Maxillary Probe Compared to References}

Table 2 shows the number of cases in which the 124 examiners correctly identified the transparency of gingival margin through the visualization of the probe.

For each examiner, the percentage of correctly classified pictures has been estimated on 53 pictures and compared with references. The mean average percentage of correctly classified pictures has been estimated for each type of examiner and whatever the type of examiner.

The percentile of agreement is greater than $70 \%$ in maxilla as well as in mandible. Whatever the group, the percentiles of agreement are similar in maxilla and mandible.

\subsection{Percentage of Correctly Classified Pictures Based on Gingival Biotypes}

For each examiner, the percentage of correctly classified pictures based on the type of pictures has been evaluated. The mean percentage of correctly classified pictures has been evaluated for each group of examiners and whatever the group of examiners with a $95 \%$ confidence interval. Results are shown in Table 3: an imbalance was observed on the maxillary pictures concerning the gingival biotype ( 9 thin for 39 thick) and on the thin biotype between maxilla and mandible ( 9 versus 23 , respectively) despite there being no statistical difference $(\mathrm{p}<0.0001)$. Concerning the thick gingival biotype, the difference is less important between mandible and maxilla. Due to the increasing number of pictures, the difference is globally masked $(\mathrm{p}<0.001)$.

The percentile of agreement is quite similar in the mandible for both thin $(74 \%)$ and thick $(70 \%)$ gingival biotypes. We find the same agreement level for thick biotypes in maxilla $(77 \%)$, but the result of correctly identified cases for thin biotypes in maxilla is only about $47 \%$.

\subsection{Agreement between Mandibular Probe and Maxilla Probe Results}

For each examiner, the percentage of agreement was eva- 
Table 2. Accuracy of mandibular and maxillary probes compared to references' classification.

\begin{tabular}{|c|c|c|}
\hline & $\begin{array}{c}\text { Mandible probe compared to } \\
\text { mandible reference }\end{array}$ & $\begin{array}{c}\text { Maxillary probe } \\
\text { compared to maxillary } \\
\text { reference }\end{array}$ \\
\hline & $\begin{array}{l}\text { Mean percentage of correctly } \\
\text { classified pictures [CI 95\%] }\end{array}$ & $\begin{array}{c}\text { Mean percentage of } \\
\text { correctly classified } \\
\text { pictures }[\mathrm{CI} 95 \%]\end{array}$ \\
\hline$C(n=9)$ & $78 \%[74 \% ; 81 \%]$ & $75 \%[71 \% ; 80 \%]$ \\
\hline$D(n=58)$ & $73 \%[71 \% ; 75 \%]$ & $71 \%[69 \% ; 74 \%]$ \\
\hline$I(n=9)$ & $75 \%[71 \% ; 78 \%]$ & $72 \%[65 \% ; 79 \%]$ \\
\hline$T(n=48)$ & $67 \%[66 \% ; 69 \%]$ & $70 \%[68 \% ; 72 \%]$ \\
\hline $\begin{array}{l}\text { Total } \\
(n=124)\end{array}$ & $72 \%[70 \% ; 73 \%]$ & $71 \%[70 \% ; 73 \%]$ \\
\hline
\end{tabular}

Table 3. Percentage of correctly classified pictures based on the characteristics of biotypes.

\begin{tabular}{|c|c|c|c|}
\hline & & $\begin{array}{c}\text { Mandible probe } \\
\text { compared to mandible } \\
\text { reference }\end{array}$ & $\begin{array}{c}\text { Maxillary probe } \\
\text { compared to maxillary } \\
\text { reference }\end{array}$ \\
\hline & & $\begin{array}{c}\text { Mean percentage of } \\
\text { correctly classified } \\
\text { [CI 95\%] }\end{array}$ & $\begin{array}{c}\text { Mean percentage of } \\
\text { correctly classified } \\
\text { [CI } 95 \%]\end{array}$ \\
\hline \multirow[t]{2}{*}{$C(n=9)$} & Thin & $83 \%[69 \% ; 96 \%]$ & $46 \%[24 \% ; 67 \%]$ \\
\hline & Thick & $74 \%[64 \% ; 84 \%]$ & $82 \%[76 \% ; 88 \%]$ \\
\hline \multirow[t]{2}{*}{$D(n=58)$} & Thin & $79 \%[76 \% ; 82 \%]$ & $54 \%$ [49\%; 59\%] \\
\hline & Thick & $69 \%[65 \% ; 72 \%]$ & $75 \%[72 \% ; 78 \%]$ \\
\hline \multirow[t]{2}{*}{ I (n = 9) } & Thin & $72 \%[62 \% ; 82 \%]$ & $47 \%[28 \% ; 66 \%]$ \\
\hline & Thick & $77 \%[68 \% ; 85 \%]$ & $78 \%[68 \% ; 87 \%]$ \\
\hline \multirow[t]{2}{*}{$T(n=48)$} & Thin & $65 \%[61 \% ; 69 \%]$ & $38 \%[33 \% ; 43 \%]$ \\
\hline & Thick & $69 \%[65 \% ; 73 \%]$ & $77 \%[74 \% ; 80 \%]$ \\
\hline \multirow[t]{2}{*}{$\begin{array}{c}\text { Total } \\
(\mathbf{n}=\mathbf{1 2 4})\end{array}$} & Thin & $74 \%[71 \% ; 76 \%]$ & $47 \%[43 \% ; 51 \%]$ \\
\hline & Thick & $70 \%[67 \% ; 72 \%]$ & $77 \%[75 \% ; 79 \%]$ \\
\hline
\end{tabular}

luated on 48 pictures. Then the percent of agreement and the Kappa statistics with a 95\% interval were evaluated for each examiner. Kappa statistics were evaluated for each examiner and then an average of Kappa statistics was performed by the examiner's class. The results are shown in Table 4. The results demonstrate a weak percentile of agreement of gingival biotypes between maxillary and mandibular anterior teeth (57\%). The same results are observed when comparing maxillar and mandibular references, as shown in Table 4. The percentile of agreement for the references was about $52.83 \%$ with the kappa value ranging from -0.2537 to 0.1978 . These results demonstrate that patients with a thin mandibular gingival biotype have in $80 \%$ of cases a thick gingival biotype on the anterior maxillary teeth. If patients have a thick maxillary biotype, they present a thin mandibular biotype in $20 \%$ of cases.

\section{DISCUSSION}

Our results demonstrated that probe test is a reliable and
Table 4. Agreement between results of mandibular and maxillary probes.

\begin{tabular}{ccc}
\hline & $\begin{array}{c}\text { Mean corroborating } \\
\text { percentage [CI 95\%] }\end{array}$ & Mean Kappa [IC 95\%] \\
\hline $\mathbf{C}(\mathbf{n}=\mathbf{9 )}$ & $58 \%[49 \% ; 67 \%]$ & $0.1682[0.0741 ; 02622]$ \\
$\mathbf{D}(\mathbf{n}=\mathbf{5 8})$ & $54 \%[52 \% ; 56 \%]$ & $0.1167[0.0884 ; 0.1449]$ \\
$\mathbf{I}(\mathbf{n}=\mathbf{9 )}$ & $60 \%[53 \% ; 68 \%]$ & $0.1634[0.0843 ; 0.2426]$ \\
$\mathbf{T}(\mathbf{n}=\mathbf{4 8})$ & $59 \%[57 \% ; 62 \%]$ & $0.1491[0.1037 ; 0.1945]$ \\
Total (n= 124) & $57 \%[55 \% ; 58 \%]$ & $0.1355[0.1132 ; 0.1578]$ \\
\hline
\end{tabular}

very simple methodology for identifying the gingival biotype on the front teeth in both arches. This study also confirms our previous results in the difference between maxillar and mandibular biotypes on the same patient.

Probe test was developed to obtain a simple and reliable method for identifying gingival biotypes in patients. In comparison with our previous study based on visual examinations, the probe test presents a higher concordance rate (greater than $70 \%$ in maxilla as well as in mandible). Based on only visual examination, the probability for identifying the correct biotype was less than $50 \%$, whatever the arch. These results were in agreement with the results of Eghbali et al. [7]. Visual inspection is a part of clinical examination where the identification of a gingival biotype is considered important because differences in gingival and osseous architectures have been shown to exhibit a significant impact on the outcome of restorative or orthodontic treatments. Nevertheless, the discrimination of gingival biotypes based on probe transparency is subject to controversy. For some authors [8], the probe test is not a useful method for identifying the gingival biotype and they demonstrate that cone beam computed tomography is a preferable methodology for determining both soft and hard tissue thickness. These results are based on measurements performed on cadaver heads. On the contrary, a clinical study by Cook et al. [9] performed on 60 patients demonstrated that periodontal biotype was significantly related to probe visibility through the sulcus. The same results were obtained by Le et al. [10] in a clinical study performed on 66 maxillary anterior teeth using a comparison between periodontal probing and cone beam computed tomography. This visual determination may appear as a simple methodology for discriminating high-risk patients that may lead to further examinations performed by a periodontologist correlating the information obtained by clinical examinations and radiographs. Kan et al. [11] also demonstrated in a clinical study performed on 48 patients that assessment with a periodontal probe is an adequate, reliable, and objective method in evaluating gingival biotypes. Their results correlated the probe test examination and direct measurement performed after a single tooth extraction in the maxillary anterior teeth region. 
Moreover, this study shows that a difference in gingival biotypes may exist between maxilla and mandible. In fact, when a thick biotype is diagnosed in the maxilla there is a thin biotype in the mandible in $20 \%$ of cases. These results suggest, in agreement with our previous study [1], that we shall make a difference between maxilla and mandible gingival identification. Few clinical studies have been performed concerning the differences between maxillary and mandibular arches. A thicker mandibular gingiva was observed by Vandana and Savitha in 2005 [5], whereas Müller et al. [3] demonstrated a thicker gingiva in maxilla when the thinnest facial gingiva was found in maxillary canines and first mandibular premolars. All these results reinforce the hypothesis of there being a difference in gingival biotypes between maxilla and mandible in the same patient. Most of the studies characterizing gingival biotypes have been performed for maxillary anterior restorations using dental implants where the esthetic success depends partially on soft tissue integration $[8,9,11]$. Mandibular arch is rarely concerned by this esthetic problem in implant therapy, but orthodontic treatment may lead to dehiscences during the labial movement of mandibular incisors or canines [12,13]. Further studies are necessary to explore and discriminate gingival biotypes in relation to the different groups of teeth on each arch.

\section{CONCLUSION}

From this study, we can conclude that the use of probe transparency through the gingival margin is an accurate and simple method for gingival biotype diagnosis to discriminate a thin gingival from a thick gingival. Additionally, individualising the gingival biotype for each of the arches appears to be a new outcome that needs further investigation. Such an approach is appropriate for orthodontic practice or implant therapy prior to dental extraction. However, it seems that individualised data on tooth gingival biotype can help orthodontists to better control and prevention of the side effects of their treatments and implantologists to improve the esthetical results of dental implant treatment.

\section{REFERENCES}

[1] Cuny-Houchmand, M., Renaudin, S., Leroul, M., Planche, L., Le Guehennec, L. and Soueidan, A. (2013) Gingival biotype assessement: Visual inspection relevance and maxillary versus mandibular comparison. Open Dentistry Journal, 7, 1-6. doi:10.2174/1874210601307010001

[2] Muller, H.P. and Eger, T. (1997) Gingival phenotypes in young male adults. Journal of Clinical Periodontology, 24, 65-71. doi:10.1111/j.1600-051X.1997.tb01186.x

[3] Muller, H.P., Heinecke, A., Schaller, N. and Eger, T. (2000) Masticatory mucosa in subjects with different periodontal phenotypes. Journal of Clinical Periodontology, 27, 621-626.

doi:10.1034/j.1600-051x.2000.027009621.x

[4] De Rouck, T., Eghbali, R., Collys, K., De Bruyn, H. and Cosyn, J. (2009) The gingival biotype revisited: Transparency of the periodontal probe through the gingival margin as a method to discriminate thin from thick gingiva. Journal of Clinical Periodontology, 36, 428-433. doi:10.1111/j.1600-051X.2009.01398.x

[5] Vandana, K.L. and Savitha, B. (2005) Thickness of gingiva in association with age, gender and dental arch location. Journal of Clinical Periodontology, 32, 828-830. doi:10.1111/j.1600-051X.2005.00757.x

[6] Kyllar, M. and Witter, K. (2008) Gingival thickness in dogs: Association with age, gender, and dental arch location. Journal of Veterinary Dentistry, 25, 106-109.

[7] Eghbali, A., De Rouck, T., De Bruyn, H. and Cosyn, J. (2009) The gingival biotype assessed by experienced and inexperienced clinicians. Journal of Clinical Periodontology, 36, 958-963. doi:10.1111/j.1600-051X.2009.01479.x

[8] Fu, J.H., Yeh, C.Y., Chan, H.L., Tatarakis, N., Leong, D.J. and Wang, H.L. (2010) Tissue biotype and its relation to the underlying bone morphology. Journal of Periodontology, 81, 569-574. doi:10.1902/jop.2009.090591

[9] Cook, D.R., Mealey, B.L., Verrett, R.G., Mills, M.P., Noujeim, M.E., Lasho, D.J. and Cronin, R.J. (2011) Relationship between clinical periodontal biotype and labial plate thickness: An in vivo study. International Journal of Periodontics and Restorative Dentistry, 31, 345-354.

[10] Le, D., Zhang, H., Hu, W.J. and Liu, D.G. (2012) Preliminary study on gingival biotype by periodontal probing. Chinese Journal of Stomatology, 47, 81-84.

[11] Kan, J.Y., Morimoto, T., Rungcharassaeng, K., Roe, P. and Smith, D.H. (2010) Gingival biotype assessment in the esthetic zone: Visual versus direct measurement. International Journal of Periodontics and Restorative Dentistry, 30, 237-243.

[12] Melsen, B. and Allais, D. (2005) Factors of importance for the development of dehiscences during labial movement of mandibular incisors: A retrospective study of adult orthodontic patients. American Journal of Orthodontics and Dentofacial Orthopedics, 127, 552-561. doi:10.1016/j.ajodo.2003.12.026

[13] Joss-Vassalli, I., Grebenstein, C., Topouzelis, N., Sculean, A. and Katsaros, C. (2010) Orthodontic therapy and gingival recession: A systematic review. Orthodontics and Craniofacial Research, 13, 127-141. doi:10.1111/j.1601-6343.2010.01491.x 